\title{
Perspektiven des skandinavischen Korporatismus - Dänemark und Norwegen im Vergleich
}

Trotz häufigem Abgesang ist der Korporatismus in Skandinavien noch immer lebendig. Er ist vor allem auch dort zu finden, wo die wenigsten Forscher ihn suchen: in sozialpolitischen Handlungsfeldern. Hier wirken kulturell und traditionell verankerte Beteiligungsstrukturen fort, die den Sozialpartnern in beiden Ländern Einfluss und Gestaltungsmacht geben - trotz auch in Skandinavien veränderter Rahmenbedingungen. Ein solcher Mesokorporatismus ist besonders stark in Dänemark ausgeprägt.

\section{Einleitung}

Mit dem Begriff „Korporatismus” werden häufig politische Entscheidungsprozesse und institutionelle Strukturen beschrieben, die nicht nur den Staat, sondern auch die Sozialpartner in die Regulierung von Arbeit und sozialer Sicherung einbeziehen, insbesondere hinsichtlich Fragen der Entlohnung. Korporatismus wurde daher als eine Alternative zu Regulierungsmodellen gesehen, die entweder rein staatsdominiert sind oder die allein auf die Kräfte des Marktes (Neo-Liberalismus) oder den Willen von Gewerkschaften und Arbeitgeberorganisationen (Voluntarismus) vertrauen. In den Ländern Europas haben sich jedoch sehr unterschiedliche Formen des Korporatismus etabliert. So zeigte sich, dass die Beziehungen zwischen Staat und Sozialpartnern in den skandinavischen Ländern zwar Ähnlichkeiten mit den nördlichen korporatistischen Kontinentalländern aufweisen. Doch die Sozialpartner in Skandinavien sind allemal stärker organisiert. Auch sind korporatistische Strukturen bei ihnen kulturell und traditionell fester verankert als bei den südlichen Nachbarn. Doch ungeachtet solcher Differenzen ordnen Wissenschaftler Skandinavien häufig in die Kategorie „korporatistische Länder in Europa" ein. Allerdings war es oftmals Schweden, das in vergleichenden Studien als Paradebeispiel herangezogen wurde und damit quasi pars pro toto für die skandinavischen Länder stand (vgl. z. B. Korpi 1983; Katzenstein 1985; Rothstein 1992).

Weit weniger Aufmerksamkeit wurde den Beziehungen zwischen Staat und So- zialpartnern in zwei anderen skandinavischen Ländern, nämlich Norwegen und Dänemark, gewidmet. Diese Lücke will der vorliegende Beitrag schließen. Im Vordergrund steht dabei die Beantwortung der Frage: Ist der skandinavische Korporatismus im neuen Jahrhundert noch lebendig? $\mathrm{Zu}$ beachten ist allerdings, dass Begriffskonstruktionen, wie die Bezeichnung,,skandinavischer Korporatismus“, bedeutende Unterschiede hinter Gemeinsamkeiten verstecken können. Deshalb ist die zweite Untersuchungsfrage vergleichender Natur und lautet: Was sind Ähnlichkeiten und Unterschiede im skandinavischen Korporatismus?

Im Gegensatz zu den meisten anderen Studien über die Systeme der industriellen Beziehungen untersucht dieser Artikel außerdem korporatistische Arrangements nicht nur in Bezug auf lohnpolitische Fragen, sondern auch im Zusammenhang mit einer Reihe von arbeits- und sozialpolitischen Themen. Auch hier kann die Mitwirkung der Sozialpartner nicht nur legitimatorisch bedeutsam sein, sondern sich auch auf die Entscheidungsfindung selbst und die Implementation getroffener Entscheidungen qualitativ auswirken.

Der Beitrag baut sich wie folgt auf: Abschnitt 2 gibt einen knappen Einblick in die Schwerpunkte der Korporatismusdiskussion. Im Anschluss wird neben einem kurzen historischen Abriss die Mitwirkung der Sozialpartner in Dänemark (Abschnitt 3) und Norwegen (Abschnitt 4) in den vergangenen Jahren beschrieben, und zwar in den Bereichen der Aktivierungspolitik, Weiterbildung und der Arbeitsmarktrenten, ${ }^{1}$ die seit einigen Jahren eine zweite Säule der Altersicherung sind. Im Schlussteil (Abschnitt 5) werden Gemeinsamkeiten und Unterschiede zwischen den beiden Län- dern im Hinblick auf die Zukunftsfähigkeit des skandinavischen Korporatismus diskutiert.

\section{Annahmen und Schwerpunkte der Korporatismusforschung}

Ihren Ursprung hat die Theorie des Korporatismus Mitte der 1970er Jahre. Während die damals vorherrschenden Pluralismustheorien davon ausgingen, dass der Staat eine mehr oder weniger neutrale Einheit sei und versuche, zwischen verschiedenen Interessen in der Gesellschaft, die alle als gleichberechtigt angesehen wurden, zu vermitteln, betonten die korporatistischen Theorien, dass der Staat nicht neutral sei. Folglich würde er den verschiedenen organisierten Interessen ungleiche Möglichkei-

\footnotetext{
In Skandinavien gibt es Grundrentensysteme, die durch die sogenannten kollektivvertraglichen Arbeitsmarktrenten ergänzt werden - eine am Äquivalenzprinzip orientierte, kapitelgedeckte zweite Säule in der Alterssicherung.
}

Mikkel Mailand, Associate Professor an der Universität Kopenhagen, arbeitet seit 1996 am Employment Relations Research Centre (FAOS). Arbeitsschwerpunkte: Beschäftigungspolitik, Neo-Korporatismus und Flexicurity. e-mail:mm@faos.dk

Übersetzt aus dem Englischen von Karin Vitols 
ten zur politischen Beteiligung gewähren. Untersuchungen über die Inhalte korporatistischer Arrangements bezogen sich in den 1970er Jahren größtenteils auf die Einkommenspolitik sowie auf Lohnverhandlungen. In den 1980er Jahren wurde die Perspektive erweitert. Analysiert wurden jetzt auch Handlungsfelder und Implementationsprozesse im politischen Sektor, im wirtschaftlichen Sektor sowie auf regionaler und betrieblicher Ebene. Einer der führenden Forscher dieser Zeit, Alan Cawson, definierte Korporatismus als ,, a specific socio-political process in which organizations representing monopolistic functional interest engage in political exchange with the state-agencies over public policy outputs involves those organizations in a role that combines interest representation and policy implementation through delegated self-enforcement" (Cawson 1986, S. 8). Eine umfassende Auseinandersetzung mit dem korporatistischen Konzept muss also über die Analyse von Lohnverhandlungen und Einkommenspolitik hinausgehen. Diese breite Definition des Konzeptes Korporatismus ist jüngst auch von Ebbinghaus (2002) und anderen übernommen worden und ist für die folgenden Analysen zum skandinavischen Korporatismus von besonderer Bedeutung.

Nachdem korporatistische Ansätze und Politikmuster gegen Ende der 1980er Jahre im Zuge der Ausbreitung des Neo-Liberalismus an Strahlkraft einbüßten bzw. real an Bedeutung verloren, begann Mitte/Ende der 1990er Jahre ihr Revival. Mehrere Studien zu den industriellen Beziehungen dokumentierten eine Rückkehr korporatistischer Arrangements. Trotz einer allgemeinen Schwächung der Gewerkschaften spielte die tripartistische Interessenaushandlung in der Sozialpolitik und bei der Regulierung des Arbeitsmarktes wieder eine wichtige Rolle. Der Fokus der Untersuchungen lag überwiegend auf nationalen ad-hoc-Vereinbarungen, den sogenannten „sozialen Pakten”. Diese sind gekennzeichnet durch einen politischen Tauschhandel zwischen Lohnzurückhaltung und einer neuen Beschäftigungspolitik, unter anderem bei der Arbeitslosenunterstützung, der aktiven Arbeitsmarktpolitik, bei Aus- und Weiterbildung sowie bei der Gestaltung der Altersübergänge bzw. Frühverrentungen. Häufig wurde die Wiederbelebung korporatistischer Strategien auch mit dem Versuch der EU-Länder, die Kriterien der Europäischen Wirtschafts- und Währungs- union (EWWU) zu erfüllen, in Verbindung gebracht. Einige dieser Studien (Pochet/ Fajertag 2000; Bacarro 2003) zeigen, dass diese tripartistischen Interessenaushandlungen zunehmend in Ländern anzutreffen waren - wie beispielsweise in Irland, Italien, Spanien und Portugal -, die eigentlich nicht die theoretischen Vorraussetzungen von Korporatismus erfüllten, weil sie keine starken Sozialpartner mit ausdifferenzierten Verbandsstrukturen hatten (Schmitter 1979). Die Analyse solcher neuen sozialen ad-hoc-Pakte bildete den Schwerpunkt der Korporatismusforschung in den 1990er Jahren. Außen vor blieb damals wie heute die Analyse des Korporatismus auf der Mesoebene, wie es Cawson (1986) gefordert hatte.

\section{Korporatismus in Dänemark}

\subsection{HISTORISCHE UND WIRTSCHAFTLICHE WURZELN}

Obwohl Dänemark relativ spät industrialisiert wurde, etablierten sich korporatistische Strukturen vergleichsweise früh: Als Ausgangspunkt gilt der sogenannte „September-Kompromiss" im Jahr 1899, bei dem Arbeitgeberverbände und Gewerkschaften einander anerkannten. Das dänische System der industriellen Beziehungen entspann sich entlang von Berufsgruppen.

Die sozialdemokratische Partei spielte seit den 1930er Jahren eine wichtige Rolle beim Ausbau des dänischen Wohlfahrtsstaates, auch wenn ihre Stärke hinter der ihrer Schwester-Parteien in den anderen skandinavischen Ländern zurückblieb. In Dänemark waren die Sozialdemokraten seit dem Zweiten Weltkrieg vor allem in Minderheitsregierungen an der Macht. Und in jüngerer Zeit kamen Regierungsbildungen immer häufiger ohne Beteiligung der sozialdemokratischen Partei zustande. So amtierten von 1982 bis 1993 die Konservativen und seit 2001 sind die Liberalen an der Macht, ohne allerdings die wohlfahrtsstaatliche Ausgestaltung Dänemarks (bislang) ernstlich infrage zu stellen.

Die wirtschaftliche Entwicklung verlief in den letzten Jahren insgesamt gut, trotz Abschwungphasen in den 1970er und 1980er Jahren. Mitte der 1990er Jahre nahm die als Ergebnis der vorangegange- nen Krise angestiegene Arbeitslosigkeit wieder stark ab. Auch blieb Dänemark von der internationalen Wirtschaftskrise am Beginn dieses Jahrzehnts verschont und angesichts einer Arbeitslosenquote von unter $3 \%$ trat sogar Arbeitskräftemangel auf. Die Einwanderung von Arbeitskräften aus den neuen Mitgliedstaaten soll hier Abhilfe schaffen, auch wenn solche Migrationsbewegungen in Dänemark langsamer vonstatten gehen als bei seinem nördlichen Nachbarn.

\subsection{SOZIALE PAKTE UND MAKROKORPORATISMUS}

Die industriellen Beziehungen in Dänemark zeichneten sich früher durch eine relativ zentralisierte Koordination und starke, konsensorientierte Sozialpartner aus. Ende der 1980er Jahre begannen Verschiebungen zugunsten dezentralisierter, branchenspezifischer Rahmenvereinbarungen ein System, das als „zentralisierte Dezentralisierung" bezeichnet wurde (Due et al. 1993). Der Staat hielt sich nun größtenteils zurück und beschränkte seine Rolle auf Schlichtung und Versöhnung. In Tarifverhandlungen griff er selten ein, sodass makrokorporatistische Arrangements bei Lohnaushandlungen und Einkommenspolitik in den Hintergrund traten. So gehörte Dänemark auch nicht zu den europäischen Mitgliedstaaten, in denen die Regierungen und Sozialpartner während der 1990er Jahre soziale Pakte eingingen, selbst wenn es Versuche gegeben hat, dauerhafte tripartistische Strukturen auf der Makroebene zu etablieren. ${ }^{2}$ Allerdings hat es in Dänemark viele andere tripartistische Initiativen auf nationaler Ebene gegeben. Sie sind Merkmal des „dänischen Modells“ der industriellen Beziehungen, das durch ein bilaterales und zentralisiertes System von Tarif-

\footnotetext{
2 Ein solcher Versuch ist das sogenannte Tripartistische Forum (Tripartite Forum) und das inm zugeordnete Statistische Komitee (Statistical Committee), das Ende der 1990er Jahre eine kurze Blütephase hatte. Auch die sogenannte Gemeinsame Erklärung (Common Declaration) von 1987, die eine Lohnzurückhaltung vorsah, kann als funktionales Äquivalent zu den sozialen Pakten anderer Länder gelten. Diese Gemeinsame Erklärung galt zunächst nur bis einschließlich 1998, wurde aber durch eine neue bilaterale Vereinbarung zur Lohnzurückhaltung, der sogenannten "Verhandlungsklima"-Vereinbarung ( "Klimaaftalen"), noch im selben Jahr verlängert. Ergänzend fanden tripartistische Dialoge bei sozialpolitischen Themen statt.
} 
verhandlungen gekennzeichnet ist, ergänzt durch die Beteiligung der Sozialpartner an der Formulierung und Implementation arbeitsmarktnaher Gesetzgebung (Due et al. 1993).

\subsection{MESOKORPORATISMUS - KORPORATISMUS IN SPEZIFISCHEN POLITIKBEREICHEN}

Korporatismus in Dänemark ist geprägt durch die Mitwirkung der Sozialpartner bei der Formulierung sozialpolitischer Politiken und deren Umsetzung. Dies wird im Folgenden am Beispiel der betrieblichen Altersvorsorge, der Aktivierungspolitik und der Weiterbildung aufgezeigt, da sich in diesen drei Bereichen einige der wichtigsten Reformen der jüngeren Arbeitsmarktund Sozialpolitik in Dänemark vollzogen haben.

\section{ARBEITSMARKTRENTEN}

Die erste Arbeitsmarktrentenversicherung (vgl. Fußnote 1) wurde 1980 in Dänemark etabliert. Allerdings wurde in den 1980er Jahren nicht mehr als ein Drittel der Arbeitnehmer von solchen Rentenprogrammen erfasst. ${ }^{3}$ Die Initiative zu einer zweiten Säule der Alterssicherung ging größtenteils auf die Regierung zurück, die das Problem der Altersarmut bekämpfen wollte. Das Interesse der Gewerkschaften war, Einfluss und Kontrolle über solche Rentenfonds zu gewinnen - eine Perspektive, die die Arbeitgeber fürchteten. Sie lehnten zentralisierte und von Arbeitnehmervertretern dominierte Fonds ab. Die Gewerkschaften gaben deshalb den Versuch auf, einen bilateralen Konsens mit den Arbeitgebern zu erreichen und nutzten stattdessen unilateralen Lobbyismus, um Einfluss auf eine erwartete entsprechende Gesetzgebung auszuüben.

Für einige Zeit schien es, als ob die Strategie des größten Gewerkschaftsbundes (Landsorganisationen i Danmark (LO)), durch Lobbyismus auf die Gesetzgebung Einfluss zu nehmen, erfolgreich sein würde. Im Anschluss an die Gemeinsame Erklärung (vgl. Fußnote 2) fanden zwischen 1987 und 1988 tripartistische Verhandlungen zu den Arbeitsmarktrenten statt. Vorgeschlagen wurden vier unterschiedliche Modelle, die entweder auf den einzelnen Arbeitnehmer, die betrieblichen Einrichtungen, die Branchenzusammenhänge oder die nationale Ebene zielten. Das erste Mo- dell wurde von der LO strikt abgelehnt, wohingegen das branchenübergreifende Modell beim Bündnis der dänischen Arbeitgeber (Dansk Arbejdsgiverforening (DA)) keinen Zuspruch fand. Einigungsversuche scheiterten, die Gesetzgebungsinitiative stockte und erst die Tarifverhandlungen im Jahr 1991 führten zur sukzessiven Implementation branchenspezifischer Rentenversicherungen in Form von Investmentgesellschaften unter paritätischer Regulierung der Sozialpartner und mit Gewerkschaftsvorsitz. Ausschlaggebend für diesen Durchbruch war zum einen die Tatsache, dass die LO zu der Auffassung gelangt war, dass die Arbeitsmarktrenten nicht durch eine Gesetzgebung eingeführt werden würde. Zum anderen hatte auch das Bündnis der dänischen Arbeitgeber (DA) seine Einstellung geändert.

Der Anteil der Arbeitnehmer, die Anspruch auf eine solche Arbeitsmarktrente haben, wie auch der Prozentsatz des Einkommens, der dem Versicherungsfond zugeleitet wird, haben in den 1990er Jahren stetig zugenommen. Schätzungen des Jahres 2003 gehen davon aus, dass $92 \%$ aller abhängig Beschäftigten Anwartschaften auf Versicherungsleistungen haben, wenn auch in unterschiedlicher Höhe. Unter Bezug auf diese Angaben sah die sozialdemokratische Regierung (1993-2001) - gestützt von der LO - nunmehr keinen Bedarf, eine zusätzliche Gesetzgebung, die noch von der konservativen Vorgängerregierung angedacht war, einzuführen. Eine solche Gesetzgebung hätte die Arbeitsmarktrenten auf alle Beschäftigungsgruppen erweitert. Bilanziert werden kann, dass die Entwicklung der dänischen Arbeitsmarktrenten eine neue Ära einleitete, die durch bilaterale Verhandlungen gekennzeichnet war - allerdings nicht gänzlich ohne aktive Beteiligung des Staates. Das System der betrieblichen Altersversorgung wurde vor allem auch ausgebaut, weil eine einseitige staatliche Finanzierung von Rentenzahlungen problematisch erschien und von einer tripartistischen Gremienarbeit andere Lösungen erhofft wurden. Allerdings plante die Regierung eine Anschlussgesetzgebung. Als Folge dieses Prozesses hat sich eine klare Teilung von Verantwortlichkeiten entwickelt, sodass der Staat nun alleiniger verantwortlicher Akteur für die Regulierung, Finanzierung und Bereitstellung der öffentlichen Altersvorsorge ist, während die Arbeitsmarktrenten in der Regie der Sozialpartner liegen.

\section{AKTIVIERUNGSPOLITIK}

Seit den 1970er Jahren wurde in Dänemark durch eine Aktivierungspolitik versucht, auf die Beschäftigungskrise zu reagieren. Von Beginn an wirkten die Sozialpartner bei der Formulierung und Umsetzung der Maßnahmen mit. Mit der Arbeitsmarktreform 1994 wurde dieser Einfluss der Verbände gestärkt. Die Reforminhalte wurden in den Jahren 1991-92 von einer den Gesetzentwurf vorbereitenden Kommission beschlossen, die mehrheitlich mit den Sozialpartnern besetzt war. Die 1993 frisch an die Macht gekommene sozialdemokratisch geführte Regierung folgte den Empfehlungen der Kommission. Die Arbeitsmarktreform stärkte den Einfluss der Sozialpartner über die sogenannten regionalen Arbeitsmarkträte (De Regionale Arbejdsmarkedsråd (RAR)). Das Konsultationsrecht (im Zusammenhang mit bestimmten Entscheidungen) der Räte wurde zum Entscheidungsrecht (Konzertierung) ausgeweitet. Auf der nationalen Ebene beriet der Rat fortan das Arbeitsministerium. Allerdings begann bereits 1996 ein Prozess, der die Aktivierungspolitik teilweise wieder zentralisierte und etwas von dem erlangten Mehreinfluss der Sozialpartner minderte. Die „Aktivierung" selbst zeichnete sich inhaltlich vor allem durch Maßnahmen wie individuelle Handlungspläne, Weiterbildung und - zu einem gewissen Grad auch - Subventionierung von Arbeitsplätzen aus (Mailand/Due 2003).

Zeitgleich zu der Arbeitsmarktreform, die größtenteils, aber nicht ausschließlich, darauf abzielte, das Verhalten von Arbeitslosen zu ändern (die Angebotsseite), wurden auch Versuche unternommen, die Unternehmen (die Nachfrageseite) stärker in die Verantwortung zu nehmen. Im Jahr 1994 wurde die Kampagne „Unsere gemeinsame Sorge - die soziale Verantwortung der Unternehmen“ („Our Common Concern - the social responsibility of companies“) gestartet. Sogenannte „Tätigkeiten zu besonderen Bedingungen” und „Flexjobs“ (vgl. Bredgaard et al. in diesem Heft) für Personen mit eingeschränkter Arbeitsfähigkeit wurden in zwei Umsetzungsformen eingeführt: In der ersten Variante wurden diese Stellen von den Kommunen verwaltet und vermittelt. Bei der zweiten be-

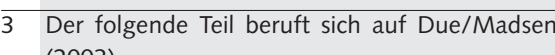
(2003). 
stimmten sogenannte soziale Kapitel in den Tarifverträgen die Konditionen. Letztere sahen jedoch - anders als bei den Kommunen - keine Lohnsubventionen vor und waren folglich weit weniger attraktiv als die vergleichbare Beschäftigungsform der Kommunen (Hohnen 2002).

Die liberal-konservative Regierung, die Ende 2001 an die Macht kam, führte eine weitere Reform der Arbeitsmarktpolitik durch. Von nun an wurde das Augenmerk verstärkt auf eine Unterstützung bei der Beschäftigungssuche sowie in einem gewissen Umfang auch auf die Berufsbildung im privaten Sektor gelegt. Die berufliche Weiterbildung als Aktivierungsmaßnahme wurde dagegen eher eingeschränkt. Zudem wurden die Aktivierungsangebote für die Bezieher von Sozialgeld (Grundsicherungsleistungen) an die Leistungen für die Bezieher von Versicherungsleistungen angepasst. Des Weiteren sind verschiedene tripartistisch oder multiparitätisch besetzte Gremien auf zentraler Ebene fusioniert worden. Die Reform ermöglichte auch den verstärkten Einsatz von neuen privaten Akteuren, wie Zeitarbeitsagenturen, privaten Weiterbildungsinstitutionen und Beratungsunternehmen, die insbesondere Angebote für Aktivierungsmaßnahmen zur Verfügung stellen sollten.

Unter der liberal-konservativen Regierung wurden die Sozialpartner zwar - wie auch unter ihren Vorgängern - punktuell in die Politikgestaltung einbezogen, doch zukünftig wird auf der regionalen Ebene, wo der Einfluss der Sozialpartner zuvor am stärksten war, ihr Einfluss geschwächt. Welche Effekte dies auf den Korporatismus in diesem Politikbereich hat, kann noch nicht abgeschätzt werden.

\section{WEITERBILDUNG}

Weiterbildung wurde in Dänemark insbesondere durch die Gründung von Weiterbildungszentren am Arbeitsmarkt (AMUs) in den 1960er Jahren populär. Im Laufe der Zeit ist eine Reihe von Weiterbildungsangeboten entwickelt worden, die nicht nur beruflich orientiert waren. Das Angebot gliedert sich in Erstausbildungen und Erwachsenenweiterbildung (bei der die AMU von besonderer Bedeutung sind) sowie in allgemeine Erwachsenenbildung und Allgemeinbildung („folkeoplysning“). Die Weiterbildung - einschließlich der Ausbildung für Arbeitnehmer - ist in Dänemark in einem höheren Ausmaß als in den meis- ten anderen EU-Ländern öffentlich finanziert. Dänemark ist zudem Spitzenreiter bei der Nutzung von Weiterbildung.

Die Steuerung erfolgt über die „berufliche Selbstverwaltung" (occupational selfgovernance) und entsprechend paritätisch oder tripartistisch besetzte Gremien existieren auf allen politischen Ebenen. Es gibt beispielsweise einen branchenübergreifenden Rat, der den Minister berät, branchenspezifische „Weiterbildungsräte” sowie das Direktorium an den einzelnen Schulen.

Mit der jüngsten Reform zur Weiterbildung aus dem Jahr 2001 wurde ein tripartistisch besetzter Ausschusses für die Finanzierung der Aus- und Weiterbildung auf dem Arbeitsmarkt eingesetzt. Er sollte den Umfang von Weiterbildungsmaßnahmen und ihre Finanzierung beraten, mit dem Ziel, zum einen die öffentliche Finanzierung der Weiterbildung auf gering qualifizierte Arbeitnehmer und formale, anerkannte Kompetenzen zu beschränken, zum anderen die Unternehmen bei der Finanzierung stärker in die Pflicht zu nehmen und gegebenenfalls sogar zusätzliche Finanzmittel von den Arbeitgebern einzufordern. Hiermit im Einklang steht auch die Tendenz, die Sozialpartner in der Weiterbildung, nämlich in Bezug auf die Einführung und Erweiterung von Rechten der Arbeitnehmer, an Weiterbildungen teilzunehmen, stärker zu beteiligen (Due et al. 2005).

Der Reformentwurf (2001) geht auf eine gesetzesvorbereitende Kommission zurück, die bereits im Jahr 1999 ihre Arbeit beendet hatte. Zwar hatten die Sozialpartner auf die Arbeit des Komitees keinen Einfluss, aber das hier erarbeitete Weißbuch wurde im "tripartistischen Forum" und dem ihm zugeordneten „Statistischen Komitee“ besprochen (vgl. Fußnote 2). Während der Verhandlungen stimmten die Sozialpartner den meisten Reformvorschlägen des Weißbuches - einschließlich der Richtlinien zur Finanzierung der Aus- und Weiterbildung auf dem Arbeitsmarkt, die sich später auch im Gesetzestext wiederfanden - zu (Due/Madsen 2006). Was also als ein Prozess begann, von dem die Sozialpartner ausgeschlossen waren, endete schließlich in Konzertierung.

Im Herbst 2004 veröffentlichte die Verwaltung dann eine umfassende Studie zur Weiterbildung in Dänemark, die Anlass für weitere tripartistische Verhandlungen $\mathrm{zu}$ Beginn des Jahres 2006 war und in der Absichtserklärung mündete, eine tief greifende Reform im Bereich der Weiterbildung anzuvisieren, um die Finanzlast der Weiterbildung zum großen Teil auf die Sozialpartner abzuwälzen. In den neuen Tarifverträgen für den privaten Sektor von 2007 wurden Weiterbildungsfonds etabliert, jedoch mit einem geringen Finanzierungsvolumen. Höhere Fondsbeiträge könnten, so die Einschätzung, immer noch in späteren Tarifrunden vereinbart werden. Im verarbeitenden Gewerbe sind seit 2008 alle Arbeitgeber verpflichtet, ca. $35 €$ pro Angestellten jährlich an den Fonds abzuführen. Dieser Betrag wird im Jahr 2009 verdoppelt. Von 2009 an erhalten zudem alle Beschäftigten mit mehr als neun Monaten Betriebszugehörigkeit das Recht auf zwei Wochen Weiterbildung nach ihrer eigenen Wahl, finanziert aus den Weiterbildungsfonds. In zukünftigen Tarifverhandlungen wird die weitere Erhöhung dieser Fondsbeiträge erwartet (Due/Madsen 2006; DI \& CO-industri 2007; Mailand 2008).

Zusammenfassend ist die Rolle der Sozialpartner hier mit derjenigen in der Aktivierung vergleichbar: Beratung und gelegentliche Konzertierung in Gesetzfindungsprozessen und dauerhafte Beteiligung der Sozialpartner bei der Umsetzung der Gesetzgebung auf allen Ebenen. Jedoch unterscheidet sich der Bereich Weiterbildung von dem der Aktivierung dadurch, dass die Einrichtung neuer tripartistischer Gremien und die Zuordnung von Verantwortlichkeiten einem stärkeren Wandel unterliegen. In Zukunft könnten auch bilaterale Beziehungen für die Regulierung der Nachfrage nach - und der Finanzierung von - Weiterbildung an Bedeutung zunehmen.

\section{Korporatismus in Norwegen}

\subsection{HISTORISCHE UND WIRTSCHAFTLICHE WURZELN}

Die Industrialisierung in Norwegen setzte spät und ungleichmäßig ein. Diese Entwicklung hat - wie in Dänemark, aber im Gegensatz zu Schweden - zu einer diversifizierten Ausprägung des Gewerkschaftswesens und zu bestimmten Klassenkoalitionen geführt.

Wie auch im dänischen Fall stellte die sozialdemokratische Partei in den 1940er bis 1980er Jahren die meiste Zeit die Regierung, wobei die Partei in Norwegen ver- 
gleichsweise stärker war. In den letzten Jahrzehnten mussten die Sozialdemokraten allerdings für längere Zeiträume in die Opposition: von 1981-1986, 1997-2000 und wieder 2001-2005. Die Beziehung zwischen dem größten Gewerkschaftsbund (Landsorganisasjonen i Norge (LO)) und den Sozialdemokraten sind allerdings noch immer sehr eng, und im Gegensatz zu der Situation in Dänemark gibt es hier auch enge formelle Verbindungen (Lismoen 2002).

Die norwegische Wirtschaft hängt auch heute noch vom Export ihrer Bodenschätze ab. Der anhaltende Konjunkturaufschwung in Norwegen - unterstützt durch die Einnahmen aus dem Export von Öl und anderen Bodenschätzen - hält die Nachfrage nach Arbeitskräften auf hohem Niveau. Die Arbeitslosenquote lag in Norwegen im Jahr 2006 bei 3,5\% (OECD 2008).

\subsection{SOZIALE PAKTE UND MAKROKORPORATISMUS}

Norwegen hat traditionell im europäischen Vergleich eine der zentralisiertesten Formen der industriellen Beziehungen und der Staat hat - im Gegensatz zur Situation in Dänemark - auch bei der Lohnsetzung im privaten Sektor eine entscheidende Rolle. Der gewerkschaftliche Organisationsgrad liegt in Norwegen niedriger als in Dänemark. Dies könnte darauf zurückzuführen sein, dass die Gewerkschaften nicht für die Verwaltung der Arbeitslosenversicherung zuständig sind wie in Dänemark.

Seit den 1970er Jahren verursachte die Abhängigkeit von den Bodenschätzen noch größere Wirtschaftschwankungen als anderswo. Dies führte nicht selten zu staatlichen Eingriffen in die Lohnpolitik. In den Jahren 1987-88 resultierte aus informellen Gesprächen zwischen den wichtigsten Sozialpartnern und der von der Arbeiterpartei geführten Regierung eine Vereinbarung als Antwort auf die Wirtschaftskrise, die fallenden Ölpreise und industriellen Konflikte. Die inflationistische Lohnspirale sollte durchbrochen und die Wettbewerbsfähigkeit wiederhergestellt werden durch eine Kombination von zentralisierter Einkommenspolitik und Austeritätspolitik.

Dieses informelle Abkommen wurde in der sogenannten Solidaritätsalternative (Solidarity Alternative) 1992 kodifiziert, einem von der Regierung errichteten tripartistischen Gremium. Ziel war es, die Arbeitslosigkeit in fünf Jahren zu halbieren durch die Kombination von Lohnmäßigung, Überprüfung des Einsatzes öffentlicher Finanzmittel, Ausweitung der aktiven Arbeitsmarktpolitik und Weiterbildung sowie durch eine Strukturpolitik und Geldmengenpolitik, die auf einen stabilen Wechselkurs zielt (Dølvik/Martin 2000, S. 280).

Die Solidaritätsalternative konnte allerdings nicht - jedenfalls in kurzfristiger Hinsicht - die Probleme der Wirtschaft beheben. Die Arbeitslosigkeit stieg auf $9 \%$ an, und die private Produktion nahm ab. Mithilfe der Einnahmen aus dem Ölexport und der Verbesserung der internationalen Wirtschaftslage fing die norwegische Wirtschaft aber ab 1993 an, sich zu erholen.

Der Wechsel von der von der Arbeiterpartei geführten Regierung hin zur konservativen Zentrums-Regierung im Jahr 1997 führte auch zum Ende der lohnmäßigenden Solidaritätsalternative. Die neue Regierung schlug die Abschaffung eines Feiertages sowie Kürzungen im Krankengeld und weiterer staatlicher Geldleistungen als Teil eines Krisenpakets vor. Dieser Vorschlag stieß bei den Gewerkschaften auf massive Kritik und führte zu dem ersten Streik in der Geschichte der norwegischen Gewerkschaftsbewegung, an dem sich alle gewerkschaftlichen Dachverbände beteiligten. Obwohl die LO die Lohnmoderation unterstützt hatte, kam es im Jahr 1998 zu großflächigen Streiks und Lohnzuwächsen in Rekordhöhe, die weit über der Inflationsrate lagen. Diese Entwicklung zeigt nach Meinung einiger Beobachter die Unfähigkeit der norwegischen Gewerkschaftsbewegung, die Politik der Lohnmäßigung bei einem lang anhaltenden Wirtschaftsboom dauerhaft durchzuhalten (Dølvik/ Martin 2000, S. 284ff.)

Die Regierung konnte gegen Ende 1998 allerdings dennoch die Gewerkschaften erneut zur Bekämpfung einer weiteren Krise einbinden. Diesmal sind auch alle Dachverbände von beiden Seiten an den Tisch verschiedener tripartistischer Gremien gebeten worden, wohingegen zuvor die LO andere gewerkschaftliche Dachverbände von Gesprächen zur Einkommenspolitik ausgeschlossen hatte. Das wichtigste dieser Gremien war die sogenannte ArntsenKommission, die die Lohntarifrunde 1999 vorbereiten sollte. In der Kommission wurde vereinbart, dass die bevorstehende Lohnsteigerung unter $4 \%$ ausfallen sollte. Die LO konnte allerdings erreichen, dass als Ausgleich für die Versprechungen zur
Lohnzurückhaltung zumindest Verbesserungen in der Weiterbildung eingeführt werden sollten (ebd., S. 286).

Vor einigen Jahren dann wurde Norwegen massiv von der internationalen Wirtschaftskrise getroffen. 25.000 Arbeitsplätze gingen in den Jahren 2002 und 2003 verloren. Die von der Zentrumspartei geführte Regierung, die 2001 nach gerade erst einem Jahr sozialdemokratischer Regierung an die Macht gekommen war, griff deswegen erneut auf das Instrument einer konzertierten Einkommenspolitik zurück. Versuche, den Arbeitsmarkt zu deregulieren, insbesondere im Hinblick auf befristete Beschäftigung, scheiterten an der Arbeiterpartei, die 2005 erneut an die Regierung kam (Dølvik 2007, S. 27).

\subsection{MESOKORPORATISMUS - KORPORATISMUS IN SPEZIFISCHEN POLITIKBEREICHEN}

Wie auch in Dänemark existieren in Norwegen in verschiedenen Politikbereichen korporatistische Strukturen - sowohl in Bereichen der Gesetzesfindung als auch bei der Umsetzung von politischen Entscheidungen.

\section{WEITERBILDUNG}

Weiterbildung ist in Norwegen seit jeher korporatistisch geprägt. Die Sozialpartner sind hier im Allgemeinen an der Politikformulierung und - durch die Vertretung in Ausschüssen - auch an der Umsetzung der Entscheidungen in 130 regionalen Lehrzentren beteiligt (European Commission 2003). Außerdem ist die berufliche Bildung Verhandlungsthema zwischen den Sozialpartnern sowohl auf Makro- als auch auf Branchenebene (Skule et al. 2002, S. 273).

Die letzte groß angelegte Reform im Weiterbildungssektor war die „Kompetenzreform“ im Jahr 1998. Sie war nicht wirklich politikfeldspezifisch, da sie zusammen mit der Lohnmäßigung verhandelt wurde. Die Reform wurde vom Gewerkschaftsbund LO initiiert, der in der Weiterbildung eine seiner Hauptaufgaben sah. Bereits 1994 vereinbarten LO und NHO (das Bündnis der Arbeitgeber) im Rahmentarifvertrag ein zusätzliches Kapitel zur Weiterbildung. 1996 beauftragte dann das norwegische Parlament die Regierung, eine Kommission zu bilden, die eine Strategie für lebenslanges Lernen vorschlagen sollte. Es wurde die „Buer-Kom- 
mission" eingesetzt, mit Beteiligung der Sozialpartner. Der Kommissionsbericht von 1997 definierte den Arbeitsplatz als wichtigsten Lernort und forderte unter anderem die Anerkennung von informell erworbenen Kompetenzen und ein Recht auf Bildungsurlaub für Arbeitnehmer. Darüber hinaus wurde eine starke Mitwirkung der Sozialpartner bei der Ausgestaltung der angestrebten Reform angemahnt, insbesondere im Hinblick auf Fragen der Finanzierung (ebd. S. 271). Die Reform wurde den Vorschlägen der Kommission entsprechend verabschiedet, ergänzt um die Gewährung von Steuervorteilen für Arbeitgeber, die ihren Beschäftigten Weiterbildungsmaßnahmen finanzieren (European Commission 2003).

Um die Reform konkret umzusetzen, wurde die Weiterbildung als Thema in den folgenden Tarifrunden aufgegriffen. In der Tarifrunde 1999 war die LO erneut bereit, Lohnzurückhaltung im Austausch für Verbesserungen in der Weiterbildung zu üben. Die Regierung versprach, 400 Mill. Norwegische Kronen (NOR) für das Kompetenz-Aufbau-Programm auszugeben. Auf ein Modell zur Finanzierung von Bildungsurlaub konnte man sich allerdings nicht einigen. In der Tarifrunde im Jahr 2000 stand das Thema Weiterbildung erneut auf der Agenda. Dieses Mal schlug die LO vor, Lohnzurückhaltung gegen die Finanzierung des Bildungsurlaubs durch die Arbeitgeber zu tauschen. Aber die verhandelnden Parteien erzielten in dieser Frage keinen Konsens und der Vorschlag der LOSpitzenvertreter konnte sich auch in einer späteren Abstimmung unter LO-Gewerkschaftsmitgliedern nicht durchsetzen. Die Mitglieder wollten sich nicht auf den Kompromiss Lohn gegen Weiterbildung einlassen, sondern sprachen sich für Lohnerhöhungen und einen höheren Urlaubsanspruch aus (Skule et al. 2002, S. 271).

\section{ARBEITSMARKTRENTEN}

Wie in Dänemark sind die Arbeitsmarktrenten eines der strittigsten Themen des letzten Jahrzehnts in der norwegischen Sozial- und Arbeitsmarktpolitik. Erst in den letzten zwei oder drei Jahren hat sich ein neues System von Arbeitsmarktrenten herausgebildet, das - anders als in Dänemark - viel stärker in den geplanten Umbau des gesamten Rentensystems integriert worden ist. Im Jahr 2001 wurde eine „Rentenkommission" eingesetzt, um die Hauptziele und Grundsätze eines einheitlichen zukünftigen Rentensystems zu skizzieren, einschließlich der Zuteilung von Verantwortlichkeiten über öffentliche und nichtöffentliche Rentenleistungen und der Möglichkeit eines fondbasierten Systems. Die Kommission - bestehend aus Experten, aber ohne die Sozialpartner - empfahl 2004 eine stärkere Orientierung des Rentensystems an der vorangegangenen Beschäftigung (Neergaard 2004). 2005 entschied das Parlament schließlich, dass alle Arbeitnehmer einen gesetzlichen Anspruch auf Arbeitsmarktrenten haben. 2003 hatten nur ungefähr $55 \%$ aller Arbeitnehmer einen solchen Anspruch (Kaar van het/Grünell 2004). Der Mindestbeitrag der Unternehmen wurde auf $2 \%$ der Lohnsumme festgesetzt. Ein Weißbuch, das sich mit anderen Rentenleistungen jenseits der Arbeitsmarktrenten beschäftigte, wurde in das Regierungsweißbuch von 2006 integriert.

\section{AKTIVIERUNGSPOLITIK}

Die Aktivierungspolitik hatte in Norwegen nie ein so starkes Profil wie in Dänemark. Die Ausgaben für die aktive Arbeitsmarktpolitik beliefen sich 2004 auf $0,4 \%$ des Bruttoinlandsprodukts (BIP) und die der passiven Maßnahmen auf 0,5\%. Im Vergleich hierzu lagen die Angaben für Dänemark bei 1,8 \% und 2,7 \% (OECD 2006, S. 271-274). Die höheren Ausgaben in Dänemark illustrieren den Versuch, die Arbeitslosenquote $\mathrm{zu}$ senken, die höher war als die in Norwegen (Halvorsen/Jensen 2004, S. 467). In Norwegen wurde hingegen in den 1960er und 1970er Jahren versucht, das Vollbeschäftigungsziel zu erreichen, indem in Krisenzeiten Regionalpolitik mit staatlicher Unternehmensgründung und der oben beschriebenen Einkommenspolitik verbunden wurde.

Gleichwohl griff auch Norwegen auf Aktivierungsmaßnahmen zurück, und als die Arbeitslosigkeit am Anfang der 1980er Jahre anstieg, wurde außerdem die maximale Anspruchsdauer für Arbeitslosenleistungen verlängert. Am Ende der 1980er Jahre änderte sich jedoch der politische Kurs und die „Arbeitslinien” forcierten Anreize und Pflichten zur Arbeitsaufnahme. Im Jahr 1998 wurde die maximale Dauer des Arbeitslosengeldes auf drei Jahre und 2003 auf zwei Jahre verkürzt (Arbeids- og Socialdepartementet 2005). Im Vergleich zur Aktivierungspolitik in Dänemark bestand in Norwegen mehr Skepsis gegen- über der Beteiligung von Arbeitslosen an Weiterbildung, subventionierter Beschäftigung und anderen „zweiten Chancen”. Stattdessen wurde stärker auf Leistungssanktionen und die Einschränkung von Leistungsansprüchen gesetzt (Halvorsen/ Jensen 2004, S. 474). Auch ist die Rolle der Sozialpartner in der Aktivierungspolitik in Norwegen nicht so stark entwickelt wie in Dänemark. Einflusslos sind sie deswegen aber nicht, aufgrund einer Reihe von tripartistischen Gremien auf nationaler und regionaler Ebene.

\section{Perspektiven des skandi- navischen Korporatismus}

\subsection{KORPORATISMUS IN DÄNEMARK UND NORWEGEN IM VERGLEICH}

Die Eingangsfrage lautete, ob der Korporatismus sowohl in Dänemark als auch in Norwegen lebendig sei. Die bisherige Analyse lässt daran keinen Zweifel. Das System der industriellen Beziehungen und die sozialen Sicherungssysteme sind zwar umstrukturiert worden, aber die Sozialpartner wurden im Allgemeinen an den Restrukturierungsprozessen und an der Umsetzung der Entscheidungen beteiligt. Insgesamt haben wir also eher Stabilität als Veränderung gefunden.

Die zweite Forschungsfrage nach Gemeinsamkeiten und Unterschieden der beiden Länder zeigt einerseits, dass es eine Vielzahl von Ähnlichkeiten gibt. Das betrifft sowohl die Strukturen als auch Aktivitäten. Erstens sind die Sozialpartner noch immer relativ stark und konsensorientiert in beiden Ländern, die - nach mehreren wirtschaftlichen Auf- und Abschwüngen nun schon seit zehn Jahren ein Beschäftigungswachstum realisieren. Zweitens haben die sozialdemokratischen Parteien trotz jüngst längerer Phasen in der Opposition - in beiden Ländern eine prägende Rolle und stehen für Gleichheitsziele. Die konservativen Regierungen haben den Wohlfahrtsstaat nicht demontiert. Sie haben ihn lediglich umstrukturiert, - aber das hatten die sozialdemokratisch geführten Regierungen auch - und sie zeigten sich auch nicht weniger als die Sozialdemokraten bereit, die Sozialpartner in politische Prozesse einzubinden. Als Drittes lässt sich außerdem festhalten, dass der Korporatis- 
mus in beiden Ländern in den hier untersuchten drei Bereichen der Arbeitsmarktund Sozialpolitik verbreitet ist, wenn sich auch Unterschiede zwischen Bereichen und Zeiträumen in beiden Ländern festmachen lassen. Es scheint auch, als ob die beiden Länder ähnliche Probleme in den drei untersuchten Bereichen der Sozialpolitik zu lösen versuchten.

Andererseits existieren erhebliche Unterschiede zwischen den korporatistischen Strukturen in beiden Ländern, die die Frage aufwerfen, ob es den skandinavischen Korporatismus gibt. Der Staat hat z. B. in Norwegen eine viel aktivere Rolle in der Lohnaushandlung gespielt als in Dänemark, und tripartistische Vereinbarungen zur Entlohnung sind in Norwegen viel weiter verbreitet. Norwegen scheint folglich eher ein „traditionell“ korporatistisches Land zu sein, also eines mit konstant korporatistischen Strukturen auf der Makroebene. Allerdings scheint der Mesokorporatismus in Norwegen in den drei betrachteten Bereichen der Sozialpolitik geschwächt und insgesamt schwächer zu sein als in Dänemark. Dieses Ergebnis wird auch von einer neueren, vergleichend angelegten Studie bestätigt, die aufzeigt, dass die Anzahl von Ausschüssen und Kommissionen mit sozialpartnerschaftlicher Beteiligung in Norwegen seit den 1970er Jahren abnimmt (Christensen et al. 2008). In Dänemark ist das nicht der Fall, weil hier Voluntarismus in den industriellen Beziehungen mit NeoKorporatismus in der Sozialpolitik verbunden wird (Mailand 2005) und der Mesokorporatismus nicht geschwächt wurde.
Dänemark stellt mit dem schwächeren Makrokorporatismus und dem stärkeren Mesokorporatismus also genau den Gegensatz zu Norwegen dar.

Es könnte noch einen weiteren Unterschied zwischen den Ländern geben, der auch mit der stärkeren Beteiligung des Staates im System der industriellen Beziehungen in Norwegen zusammenhängt. In Dänemark weist der Korporatismus viele informelle Bestandteile auf, wo die bilaterale und tripartistische Koordinierung in Bezug auf das System der industriellen Beziehungen nicht in formellen korporatistischen Gremien stattfindet, sondern außerhalb von ihnen. Die stärkere Beteiligung des Staates im System der industriellen Beziehungen und die formelleren Institutionen in Norwegen könnten dagegen auf ein anderes Gleichgewicht zwischen formell und informell im norwegischen Korporatismus hindeuten - allerdings lässt sich aus der obigen Analyse nicht eindeutig feststellen, ob dies wirklich der Fall ist oder nicht.

\subsection{ZUKUNFTSPERSPEKTIVEN}

Zum Abschluss stellt sich die Frage, ob der Korporatismus in den beiden Ländern eine Zukunft hat. Im Gegensatz zur Situation im Rahmen von sozialen ad-hoc-Pakten in Ländern ohne starke Sozialpartner erscheint es schwierig, sich den skandinavischen Korporatismus ohne starke Verbände vorzustellen. Die Mitgliederzahlen sinken in beiden Ländern nur langsam, und die Sozialpartner haben in den meisten Politikbereichen immer noch einen privile- gierten Zugang zur Regierung, selbst in Bereichen, wo ihr Einfluss im Vergleich zu früher abgenommen hat. Die Regulierung von Löhnen und Arbeitsbedingungen ist auch nicht minder Gegenstand von Verhandlungen mit den Sozialpartnern als in den letzten Jahrzehnten. Herausforderungen wie Unternehmens- oder Produktionsverlagerungen, demografische Veränderungen, Regierungswechsel, verschiedene Formen zunehmender Vermarktlichung, wirtschaftliche Auf- und Abschwünge und - insbesondere in Dänemark - Bestrebungen der Unternehmen, das System der industriellen Beziehungen zu dezentralisieren, haben zwar das etablierte Regulierungsmodell verändert, aber nicht demontiert. Außerdem wurden korporatistische Arrangements nun auch auf neue Gebiete, wie z. B. die Arbeitskräftemigration, ausgeweitet.

Trotz des teilweisen Abbaus korporatistischer Strukturen ist der Korporatismus also immer noch von Bedeutung für die Regulierung von Arbeit und sozialer Sicherung in Skandinavien. Es ist davon auszugehen, dass der skandinavische Korporatismus auch weiterhin bestehen bleibt, wenn auch nicht unbedingt gleich für ein weiteres Jahrhundert, so wenigstens im kommenden Jahrzehnt. Wenn sich allerdings der gegenwärtige langsame Trend eines rückläufigen gewerkschaftlichen Organisationsgrades in Dänemark und Norwegen verstärken sollte, dann könnte die $\mathrm{Zu}-$ kunft des skandinavischen Korporatismus auch nochmals ganz anders, also weit weniger perspektivenreich, aussehen.

\section{ITERATUR}

Bacarro, L. (2003): What is Dead and what is Alive in the Theory of Corporatism?, in: British Journal of Industrial Relations 4, S. 683-706 Cawson, A. (1986) (Hrsg.): Organized Interests and the state. Studies in Meso-corporatism, London

Christensen, P. M./Nørgaard, A. S./Rommertvedt, H./Svensson, T./ Thesen, G./Øberg, P. O. (2008): What is Corporatism, and Why Should We Care?, With Examples form Scandinavia. Unpublished paper Christensen, P. M./Nørgaard, A. S./Sidenius, N. C. (2004): Hvem Skriver Lovene? Interesseorganisationer og politiske beslutningsprocesser, Aarhus DI \& CO-industri (Central Organization of Industrial Employees in Denmark) (2007): Organisationsaftale om industriens kompetenceudviklingsfond
Due, J./Madsen, J. S./Jensen, C. S. (1993): Den danske Model - En historisk sociologisk analyse af det danske aftalesystem, København Due, J./Madsen, J. S. (2003): Fra magtkamp til konsensus - arbejdsmarkeds pensionerne og den danske model, København Due, J./Madsen, J. S. (2006): Fra storkonflikt til barselsfond - Overenskomstforhandlingerne 1998, 2000, 2004. København

Due, J./Madsen, J. S./Kolstrup, A. (2005): Overenskomsternes bestemmelser om efteruddannelse mv, FAOS, Sociologisk Institut, København Dølvik, J. E./Martin, A. (2000): A Spanner in the Works and Oil of Troubled Waters: The divergent Fates of Social Pacts in Sweden and Norway, in: Fajertag G./Pochet, P. (Hrsg.): Social Pacts in Europe - New Dynamics, Brussels: ETUI, S. 279-319. 
Dølvik, J. E. (2007): The Nordic regimes of labour market governance: From crises to success story. FAOS rådsprogram 2006-08, Oslo Ebbinghaus, B. (2002): Varieties of Social Governance: comparing the social Partners' Involvement in Pension and Employment Policies, Max Planck Institute for the Study of Societies, Cologne

European Commission (2003): Implementing lifelong learning strategies in Europe: Progress report on the follow-up to the 2002 council resolution, Norway, Bruxelles

Halvorsen, R./ Jensen, P. H. (2004): Activation in Scandinavian Welfare Policy. Denmark and Norway in a comparative perspective, in: European Societies 4, S. 461-483

Hohnen, P. (2002): Aftale baserede skånejob - en kvantitativ analyse, Socialforskningsinstituttet, København

Katzenstein, P. J. (1985): Small States in World Markets - Industrial Policy in Europe, Ithaca

Kaar van het, R. H./Grünell, M. (2004): Occupational pensions and industrial relations, EIROonline, download: www.eiro.eurofound.eu.int Korpi, W. (1983): The Democratic Class Struggle, London Lismoen, H. (2002): 2001 Annual Review from Norway, EIROnline, download: www.eurofound.eu.int

Lindvall, J./Sebbring, J. (2005): Policy Reform and the Decline of Corporatism in Sweden, in: West European Politics 5, S. 1057-1074

Mailand, M./Due, J. (2003): Partsstyring i arbejdsmarkedspolitikken perspektiver og alternativer, in: Petersen, L./Madsen, P. K. (red.): Drivkræfter bag arbejdsmarkedspolitikken, København, SFI, S. 202-233

Mailand, M. (2005): The involvement of Social Partners in Active Labour Market Policy - do the Patterns fit Expectations from Regime Theories?, in: Bredgaard, T./Larsen, F. (Hrsg.): Employment Policy from Different Angels, Copenhagen
Mailand, M. (2008): Regulering af arbejde og velfærd - mod nye arbejdsdelinger mellem staten og arbejdsmarkedets parte, København Neergaard, K. (2004): Controversial pension reform proposed. EIROnline, download: www.eirofound.eu.int

OECD (2006): Employment Outlook, Paris

OECD (2008) Factbook 2008: Economic, Environmental and Social Statistics, Paris

Pochet, P./Fajertag, G. (2000): A New Area For Social Pacts in Europe, in: Fajertag G./Pochet, P. (Hrsg.): Social Pacts in Europe - New Dynamics, ETUI, Brussels, S. 9-40

Pontusson, J. (1997): Between neo-liberalism and the German model: Swedish capitalism in transition, in: Crouch, C./Streeck, W. (Hrsg.): Political Economy of Modern Capitalism. Mapping Convergence \& Diversity, London

Rothstein, B. (1992): Den Korporativa Staten: Interesseorganisationer och Statsforvaltning i Svensk Politik, Stockholm

Schmitter, P. C. (1979): Still the century of Corporatism, in: Schmitter P. C./Lembruch, G. (Hrsg.): Trends towards Corporatist Intermediation, London

Siaroff, A. (1999): Corporatism in 24 industrial democracies: Meaning and measurement, in: European Journal of Political Research 36, S. 175205

Skule S./Stuart, M./Nyen, T. (2002): International briefing 12: Training and development in Norway, in: International journal of Training and Development 6, S. 4 